\title{
SUJETOS AL MOVIMIENTO: Canales, hitos y umbrales desde los que observar
}

\author{
Álvaro ALCONADA ROMERO \\ Universidad de Lleida \\ alvaro_alconada@hotmail.com
}

\section{SUBJECTS TO MOVEMENT: Channels, milestones and thresholds to observe}

Resumen: Las conexiones hoy vencen continuamente las fronteras físicas y humanas, y quizá sea en éstas donde consigamos materializar el trabajo etnográfico, viendo cómo se reinterpreta la vida cuando entendemos que cambia de contexto. Los conceptos de movimiento o de conexión son incomprensibles si no se entiende que hay más de un lugar o persona que además se transforman con el tiempo. Por tanto es a partir de esas dimensiones de globalidad y localidad, de identificación y adaptación, donde podemos aprehender la esencia de cualquier fenómeno social. El contexto de los movimientos de personas en África Austral ayudará a centrar estas reflexiones a través de un ejemplo que, por su constancia en el tiempo y la diversidad de circunstancias que lo han acompañado, nos ayudará a pensar el abordaje del estudio del movimiento, la presencia, el vínculo, la pertenencia o incluso los canales y vías de comunicación utilizados por poblaciones transnacionales conformando su forma de vida.

Abstract: Today connections move beyond material and human borders, and maybe we could find in them the way to do ethnographic research attending to the way in which life is performed when the context changes. It is impossible to understand the concepts of movement or connection if we do not understand first that there is more than a single place or person and that they are also transformed in the march of time. So in globally and locally dimensions, from the identification and the adaptation, is where we can achieve the essence of any social phenomenon. The context of people movements in Southern Africa helps to focus these reflections in a situation that shows different circumstances over a long time and will help us to face the study of movement, presence, links, belonging or even ways of communication used by transnational populations in their way of life.

Palabras clave: Fluir. Transición. Conexiones. Redes sociales. África Austral Flow. Transition. Connections. Social Networks. Southern Africa 


\section{Introducción}

Durante el trabajo etnográfico o bien se parte de la artificialidad de delimitar un área de estudio o bien se llega a esa idea a través de la práctica, pero esta parte de creación, delimitación e identificación, aunque necesaria para su finitud, será siempre artificial a pesar de sustentarse en una justificación que la fundamente. Paralelamente, la reflexión antropológica incluye esa continua observación local y los innumerables vínculos globales para intentar comprender y explicar lo local como parte de un todo, y lo global como síntesis de diversidades. Por ello la antropología viene acompañada de cierta tensión al tener que delimitar su objeto -población- de estudio; por moverse entre lo local y lo global respondiendo ambos universos a una misma población; e incluyendo ambos la posición del investigador como parte de ellos tanto en su relación como en su producción. De esta forma el movimiento adquiere protagonismo en el estudio y participación en sociedades dinámicas. Este campo en movimiento se aprehende a través de redes multisituadas, incluso virtuales, de vínculos de pertenencia y de una territorialidad que generalmente tiene más de social que de geográfica.

Así fue como mi investigación se fue acercando a la sociedad del sur de Mozambique ${ }^{1}$ según se expandía por redes multisituadas que desbordaban unas fronteras mientras redefinían otras: se centraba en el movimiento, sus hitos, sus intercambios y transformaciones... y lo acompañaba. Aspectos como la vivencia y los significados del origen en términos locales y globales, geográficos, comunitarios o nacionales, como interacción, como atribución y como posición en determinados contextos, da una riqueza al análisis en la que veo necesario perderse para luego acercarse con humildad en nuestras conclusiones. Los periodos de cambio y transición dentro de este flujo de ideas y personas configuran y reconstruyen la experiencia dentro de las constricciones y facilidades del contexto en que se desarrolla. En el presente texto intentaré explicar y trasladar todas esas adaptaciones a un campo diverso, multisituado y cambiante en las que, como no podía ser de otra forma, la vivencia del investigador también lo es, para intentar una delimitación razonable en la que ubicar nuestras descripciones.

Por tanto, a lo largo del presente artículo, nos iremos a esa concepción de la sociedad en movimiento y con múltiples conexiones desde tres perspectivas que se traducirán en epígrafes que podríamos avanzar de la siguiente manera: a modo de introducción presentaremos una reflexión sobre la concepción dinámica de la sociedad, materializada tanto en la construcción de espacios de intercambio y conexiones que se superponen, como en la importancia de la identificación de fronteras físicas y simbólicas desde las que se piensa y reconstruye.

En un segundo epígrafe nos adentraremos en el ejemplo de las poblaciones changana del sur de Mozambique, que integran el movimiento y fortalecimiento de las redes sociales por las que transitan con la referencia al lugar de origen. En su estudio destacaremos los "hitos" de dicha movilidad, el encuentro y la búsqueda de innovación dentro esa estrategia de continuidad como características de una red social de flujos e intercambios múltiples.

A continuación, a partir del mismo ejemplo, nos centraremos en el espacio liminal como

\footnotetext{
1 Desde 2005 he realizado trabajo de campo en las zonas rurales del sur de Mozambique, conviviendo y siguiendo una población que integra en la cotidianidad el movimiento por redes sociales que abarcaban espacios transnacionales, principalmente entre el sur del país y el este de la vecina República Sudafricana. Dicha etnografía constituyó mi tesis doctoral en 2013 (http://eprints.ucm.es/23814/) y perseguía el objetivo de mostrar la migración como parte de la reproducción de la vida local. La región viene siendo hasta hoy objeto de mi interés, mis estudios y mis publicaciones. Quiero aprovechar este espacio para agradecer las sugerencias e implicación de las coordinadoras del monográfico al que pertenece el presente artículo.
} 
transición también en lo espiritual y lo cotidiano. Lo reivindicaremos como lugar de intersección de perspectivas y de conocimiento.

Finalmente, acabaremos exponiendo las conclusiones y sugerencias metodológicas de este recorrido.

\section{Movimiento, dispersión, conexión}

Quizá parezca muy lejano el año 1987 en que Kopytoff analizaba la idea de "tribu" y cómo surgió en la sociedad europea para proyectar sobre las colonias una visión estática, primitiva e idealizada sobre lo que es un pueblo. Pero veo aún necesaria esa aclaración, no sólo en la sociedad general en que dicho concepto sigue teniendo arraigo, sino en ámbitos académicos que, si bien incorporan otros como etnia o pueblo, lo hacen desde un referente primitivo, localizado y homogéneo (al menos en mayor medida de la que se clasificarían ellos mismos). Este idealismo -en un sentido tanto peyorativo como romántico- proyectado difiere de la propia experiencia en occidente para fabricar la idea en lo ajeno de un origen común racial, cultural, psicológico, costumbrista, lingüístico... enraizado por vínculos de parentesco amplio en un lugar concreto, y que justificaría la idea romántica de nación y sus fronteras.

Esta proyección, en muchas sociedades, continúa sirviendo de justificación para la mediación en los procesos de desarrollo y modernización -entendidos como progreso lineal-, que a la vez homogeniza y limita las raíces históricas de las poblaciones a su designación fronteriza o vínculo genético. Crea la idea de continuidad e inmovilidad en grupos que realmente se han generado a partir del movimiento, el intercambio y el mestizaje, como es el caso de la inmensa mayoría de grupos humanos en el globo, siendo realmente una excepción los que han vivido un aislamiento algo más continuado como el que parecían mostrar los propios orígenes de la antropología.

Aunque herederos de esa tradición, los estudios van asumiendo que incluso la idea del surgimiento, en un momento dado, de una nación uniforme instalada en unas fronteras fijas, únicamente avanza en paralelo a una realidad preexistente de sociedades caracterizadas por el movimiento y mestizaje, debido a sus circunstancias (catástrofes, migración, guerras...) y a sus propias instituciones tradicionales (matrimonio, escisión, acogida-integración...). Y que hoy en día, con el desarrollo de las comunicaciones, estas conexiones se pierden en flujos mucho más intensos y difíciles de ubicar.

Pero el territorio sigue teniendo una función básica, que podemos entender desde el concepto "paisaje cultural" en su naturaleza material y simbólica (Felipe, 2016), como contexto socialmente creado, el lugar geográfico vinculado y en interacción con sus habitantes, definido y transformado por ellos. Así pues el territorio se construye geográfica y socialmente, y es también el lugar en el que se transita, hay una población en movimiento dentro de él que se vincula por redes a otros lugares. Papa Sow (2007: 139), tomando la idea de "nuevas diásporas africanas" de Carter (2003), nos presenta la diáspora como perteneciente a redes socio-culturales, lazos que vinculan lugares híbridos y múltiples que se reconfiguran en el movimiento y el intercambio de diferentes grupos, no necesariamente ligados a un exilio o huida, sino a una experiencia de construcción personal dentro de un grupo flexible que ocupa un territorio no delimitado por fronteras institucionales.

Así, a la referencia del territorio como origen identitario en continua evolución, también hay que añadir el territorio que se extiende paralelamente a través de nexos y conexiones; al menos así lo he podido ver en mi trabajo de campo entre las redes transnacionales que por generaciones abarcan un territorio transnacional entre diferentes puntos de Sudáfrica y Mozambique que va variando en función de las propias conexiones y las oportunidades que éstas brindan para el asentamiento, el trabajo, la invisibilidad legal, etc. Manteniendo 
también el fuerte vínculo al lugar de origen (a pesar de sus notables transformaciones y diferentes delimitaciones) pero extendiendo lo propio allá donde se instala la red social.

Desde esta idea, que ya anuncia la mundialización como elemento paralelo a esos movimientos (Sow, 2007: 136), en los últimos años han cambiado bastante las formas de comunicarse y de desplazarse. Los flujos y las redes permanecen ligadas a los territorios que transitan y se proyectan idealmente, pero además se manifiestan en el universo digital paralelo que circula por las mismas. Los que trabajamos en ciencias humanas tenemos que ser conscientes y adaptarnos a estas realidades que, al mismo tiempo que plantean un desafío a nuestros métodos, también presentan ventajas -precisamente derivadas de esta característica de desubicación-. Así, en 2014 tenemos ejemplos de los pasos de una etnografía en el continente africano en estos términos con la publicación de Smith y Mwadime (2014), basada en un intercambio de emails entre dos colegas y amigos: un antropólogo titular en EEUU y su colaborador desde Kenia, y que además incluye referencias a otros lugares del continente africano con los que ambos están ligados. La lectura del libro muestra lo revelador que puede ser mantener este tipo de vínculo para actualizar y entender el tipo de relaciones y movimientos que se producen en una y otra sociedad. En este caso, entiendo este tipo de medios como indispensables -tal como son en la vida de gran parte del mundopero no excluyentes de la presencia y aprehensión propia del campo de estudio, sin la cual no estableceríamos una relación cercana ni entenderíamos el prisma de esa(s) persona(s), el lenguaje que utiliza (el idioma y su interpretación), el significado de los hechos que nos relata, los tabúes y significados ulteriores que quizá oculte, etc.

Pero eso no es todo, porque el vertiginoso ritmo de la tecnología y la evolución de la comunicación -aunque de alcance desigual- llega cada vez a más gente evolucionando en sus formas. Por ello, hoy ya es más complicado encontrar una relación informal natural a través de emails bien estructurados, descriptivos y bien redactados que nos permitan integrarnos mejor o peor en los significados siguiendo su argumentación lineal. En este momento se requiere aún mayor interpretación, ya que la manera de estar en la lejanía y participar del grupo es a través de pequeños tweets, mensajes de chat, el idealizado muro del Facebook, Instagram, los grupos de WhatsApp... que si bien son una continua fuente de información, cuestionamiento y comunicación, también exigen una decodificación cultural y contextual que justifica un trabajo previo y paralelo en el campo.

Por otra parte, el mundo digital nos permite un contacto continuado más allá del periodo del trabajo de campo, podemos ver el contexto y las condiciones que se generan más allá de un momento temporal, cuestionar nuestras apreciaciones parciales a lo largo del tiempo y en las reacciones frente a ellas, interpretar esas relaciones para volver al campo a verlas con la familiaridad del que no se ha acabado de ir. Así mismo, mantener activos los vínculos y extenderlos por redes en las que nos podemos mover con facilidad para establecer el contacto deseado... que, en definitiva, es al mismo tiempo enriquecer y facilitar el trabajo de campo, y cumplir con su función de adaptación a la vida y formas de interacción que ya son habituales en muchas personas.

Actualmente el ritmo de plazos y resultados guía los tiempos de la investigación tanto o más que el propio ritmo de inserción y acceso al conocimiento. Ese tiempo necesario en que el etnógrafo llega a ser parte de lo que observa, como dice María Cátedra (2017: 208), y que a veces queda relegado a la urgencia de los resultados. En estos momentos en que prevalece el pragmatismo la mayor limitación suele ser la temporal, pero el momento en que vivimos también nos da las herramientas para superar ese límite y nos interpela para continuar siendo parte en la distancia. Para vivir esa parte tan cotidiana e interpretable como la presencia física.

Así, creo que el "campo virtual" no es sólo una opción, sino una realidad que se puede ver como objeto de estudio y como medio para la aplicación de determinadas técnicas (cuestionarios, entrevistas online, etc.) pero, en el caso de la etnografía, sobre todo como 
una parte de la realidad que no podemos dejar de lado en nuestra búsqueda holística de una subjetividad fundamentada. Su importancia dependerá del peso que tenga en el contexto que estudiamos y su interpretación dependerá del mismo, por lo que no creo que pueda sustituir el trabajo etnográfico sobre el (los) terreno (s).

\section{Realidad multisituada y las transiciones como objeto de estudio en África Austral}

En mi caso, el hecho de estudiar una población en movimiento no fue tanto una elección como la "abducción" por parte de una realidad que me interpelaba. Y es que el hecho de conocer "por accidente" una migración generalizada desde el lugar de partida no sólo me trajo un prisma inicial diferente, sino también un abordaje particular de un fenómeno que se constituía en el movimiento y en la conexión con diferentes nodos de la red que construía ese propio origen y la sociedad vinculada a él. Frente a la habitual visión acotada de mostrar los efectos de la migración en los lugares de destino donde se asientan poblaciones como minoría estereotipada (McDonald, 2000: 1-3), el entender la sociedad changana como comunidad transnacional a través de sus redes socio-familiares me invitaba a entender el flujo como una forma de construcción del individuo y de la sociedad a través de sus vínculos y los hitos por los que pasaba. Esos hitos que nos hablan de discontinuidades, de cambio en la rutina, de dificultades, barreras, ayudas, replanteamientos o amenazas, pero dentro de las fronteras de lo propio.

Así, partiendo de ese origen social y simbólicamente importante vimos cómo esa pertenencia local se inserta y evoluciona en el movimiento, no sólo a través del intercambio con otros lugares (también propios o apropiados), sino en las personas que fluyen y que ven en el regreso a ese origen el destino final de esa ciclicidad (Goody, 1971) que reproduce la vida en este particular movimiento: siendo la juventud el momento de viajar, visitar y fortalecer vínculos mientras sustenta a la siguiente generación que le dará relevo, y completará el ciclo pasando a una senectud ligada de nuevo a esa tierra de origen donde acabarán siendo enterrados, convirtiéndose en una parte permanentemente presente en la vida de su linaje.

Ese territorio que supone el referente identitario tiene, sin embargo, fronteras sociales y geográficas bastante difusas: la historia política y bélica reciente ha transformado el paisaje, la vida, las pertenencias y ha ido incorporando diferentes niveles de identificación con el mismo: nacional, vecinal, religioso, familiar... Paralelamente ha venido siendo afectado por las transformaciones asumidas por la comunidad en su contacto con otras realidades, su evolución, el desarrollo de los medios de comunicación y transporte, etc. Pero también por los propios movimientos tradicionales con motivo de uniones familiares, la creación de nuevos hogares, los vínculos, la transformación de las instituciones y su adaptación a nuevas formas de vida (por ejemplo el progresivo abandono de la poligamia y de la ganadería por parte de las familias campesinas y migrantes, así como la creciente incorporación de las mujeres en la empresa migratoria) e incluso el traslado del lugar de culto cotidiano acompaña los movimientos familiares en esa adaptación a nuevas circunstancias.

Pero, lejos de suponer un problema, el movimiento es parte de la evolución y nos muestra la esencia, los significados más allá del objeto físico. En la transformación vemos las fuerzas que la guían, los anhelos, y en las fronteras encontramos el cuestionamiento de lo que somos. Son los hitos los lugares y momentos en que nos detenemos y reconducimos nuestro camino evaluando nuestro origen, nuestra pertenencia y nuestros objetivos; los nodos desde los que se intercambia y se establecen relaciones por una red social que, en este caso, se construye por su vínculo con el origen desde el que nos identificamos.

Siendo el movimiento algo perceptible en el día a día de estas poblaciones, en las presencias y ausencias de sus miembros y en la llegada y envío de encomiendas, me parece interesante detenernos en el sentimiento más íntimo y sentido de vínculo espacial. Aquel que tiene que ver con la pertenencia, que se produce a través del vínculo social y el alcance 
de sus instituciones como representación conjunta que nos afecta como grupo. Se vinculan al territorio por la idea espiritual de propiedad, entendiendo que el territorio lo detentan los antepasados que vivieron en él y que, por tanto, o estamos emparentados y nos ceden el derecho de vivirlo, o debemos transitar por él con respeto y pidiendo permiso a los detentores legítimos para asegurarnos que no se torne hostil.

El territorio se identifica como propio si otras personas con las que estamos vinculados nos precedieron pasando por él, conviviendo y creando un vínculo que forma parte de la historia del lugar. La invitación y acogida, la visita, el respeto y la convivencia legitiman también la presencia y la pertenencia. Así, los caminos transitados por generaciones de migrantes son parte de lo propio y en los lugares que se detienen encontramos el vínculo y, por tanto, el intercambio y la identificación. La suerte que se pide para el viaje tiene que ver con ese encuentro con lo propio. Podríamos recurrir a la historia lejana para explicarlo, pero es la continuidad de la estrategia y forma de vida seguida por generaciones la que le da legitimidad a través de sus redes sociales. Son caminos y lugares conectados con el origen, influidos, creados y vividos a través de las noticias y las historias de parientes que pasan o se detienen en ellos, y es en ese movimiento de la población que se refuerzan y recrean conexiones con esas personas que salieron hace tiempo, es un reencuentro. Y es en el encuentro donde se manifiesta lo propio, donde aparece la ayuda y el vínculo a pesar de la distancia o la transformación que haya vivido la persona. Porque todos esos cambios aparentes en personas que se identifican con rasgos comunes nos dejan entrever más claramente lo que se mantiene, con lo que se definen y la manera en que se transforma con el tiempo.

Por esta naturaleza hablamos de lugares híbridos, que integran y comparten diferentes redes e identificaciones múltiples, en esa perspectiva que integra el pasado como precedente homogeneizado de un presente que se renueva en los bordes híbridos de la cultura (Bhabha, 1996). Son experiencias inclusivas que nos traen diferentes significados del lugar y múltiples pertenencias de las personas. Un panorama difícil de delimitar más que en la referencia lejana y particular que tiene que ver con la llegada, con ese momento de cambio, de transformación. Momento que nos lleva a través de los referentes y las circunstancias y en los que las fronteras vienen a ser pasos importantes en el cuestionamiento de la empresa y en la actitud que se debe adoptar hacia la misma. Como ya he descrito en otras ocasiones, si bien los hitos son colectivos, la vivencia y el significado también es determinado particularmente, y así la frontera puede ser lugar de tránsito (fácil o difícil), oportunidades o asentamiento (Alconada, 2013) y a menudo, cada persona juega su baza poniendo en juego recursos y estrategias personales para intentar superar los desafíos. Pero también encontramos diferentes tipos de asociaciones y estrategias colectivas para favorecer cualquiera de las finalidades.

Los periodos de transición o viajes de uno a otro lado configuran y reconstruyen la experiencia interaccionando con las propias experiencias en los lugares y las posibilidades que se abren, por ejemplo: tras una migración costosa se planteará mucho volver a cruzar la frontera salvo que encuentre un motivo de peso, también será considerado un luchador que ha vencido la adversidad, etc. Y eso hará parte de la construcción colectiva del lugar, serán anécdotas transmitidas en según qué contextos que los irán reconfigurando. En este sentido, el trabajo de campo no sólo recoge la experiencia de la observación participante, también la elaboración de discursos y calificaciones. Así la experiencia de la separación de personas queridas se suele vivir en paralelo a la experiencia de la comunicación en la distancia, a través de canales que han ido cambiando y digitalizándose, pero que también han cambiado en la naturaleza y calidad descriptiva de su discurso: mientras que investigaciones previas hablaban de cintas-casetes grabadas que se enviaban de un lado a otro en encomiendas, o de cartas escritas y leídas por determinadas personas letradas (Covane, 2001); en mi caso ya llegué con el uso extendido del teléfono fijo de la cantina, que reunía conversaciones entre los que por ahí rondaban y los que acababan de hablar con sus familiares que les contaban sus experiencias en Sudáfrica. Ya habían llegado los primeros móviles traídos 
por los migrantes, que se exhibían mientras buscaban cobertura subidos a algún murete en determinados puntos de la aldea. Hoy ya han llegado los de última generación, quedó atrás la curiosidad por las primeras fotos que hacía o las peticiones para que hiciese un video de sus ceremonias cuando vieron el resultado de mis primeros documentales... Las oportunidades son distintas, los espacios se comunican y vinculan por diferentes canales, aunque permanece la esencia que tenía tanto la cinta y el primer saco de arroz enviado desde una ciudad minera sudafricana, como el whatsapp que envía un muchacho desde un suburbio de Durban al móvil que ha regalado a su madre: estar presente.

Esa presencia no sólo incluye al hijo que salió, sino a las circunstancias que transforman su vida actual y trae consigo. Al seguir redes locales extendidas por un amplio territorio gana presencia y fortaleza en la comunidad, reactiva relaciones que no sólo le darán poder y opciones en el presente, sino que también le irán dando representación y reconocimiento dentro de los afines en el futuro. Así, la migración es un periodo de encuentro, de esfuerzo y de conocimiento de todos aquellos que forman parte de la comunidad para volver con ese vínculo a establecerse en el origen con un poder legitimado.

\section{El conocimiento como espacio liminar}

Manteniendo nuestro foco en estas sociedades que integran el movimiento en su forma de vida, podemos mantener que la cultura, la espiritualidad o las tradiciones son parte y acompañan a las personas, por ello son integradas en este flujo. En ese sentido, cuando en este caso pretendemos mirar para el pasado para hablar de la "identidad tradicional", ésta se asume como heredera de los pueblos que en sus luchas, desplazamientos, intercambios y mestizaje la constituyen y, muy importante: aún siguen manifestándose y recreándose en la manera en que son y nos hacen (Honwana, 2003). Este hecho quizá sea determinante para entender que los antepasados Tsonga, Nguni y Ndau, siguen presentes tanto en su pureza como en su mestizaje, así como lo hacen en la historia particular del lugar las luchas y los intercambios con árabes o europeos, o las conexiones con los diferentes lugares por donde se extienden los vínculos.

En este sentido asistimos continuamente a la reconfiguración de esa espiritualidad integradora, actualizada con lo que somos: tanto herederos como agentes. Los líderes espirituales y médicos tradicionales no son ajenos a estos movimientos, como conviven y no son categorías diferenciadas en el día a día de las comunidades, lo viven, y veremos que es importante que así sea. Y, por supuesto, son también caminantes y creadores de estas redes que configuran lo propio y se mueven entre esas realidades, flujos y umbrales que veníamos describiendo. Su vínculo con la tierra, su conocimiento de la naturaleza y lo local se combina con el de esos vínculos multisituados.

Pero hablemos más allá de los límites físicos, porque los médicos tradicionales, holísticos o curanderos, no sólo se detienen en estos hitos del cuestionamiento entre diferentes tipos de pertenencia, sino que esa posición de cuestionamiento y de moverse en las fronteras es la que les identifica y de la que se derivan sus capacidades. El curandero sirve de canal y de mediador entre el mundo espiritual y terrenal, y esta facultad no es un ejemplo aislado en el carácter espiritual de los curanderos del sur de Mozambique, sino que encontramos esta pertenencia ambigua en otros lugares como las vistas en representaciones en África Occidental como el origen mítico de los griots o las figuras en forma de pájaro que vuela entre dos mundos... y probablemente estos roles de mediadores están presentes en la materialización de todas las religiones. Pero en el caso del curandero, esta capacidad de ser vehículo entre realidades que le confiere la "invitación" y el vínculo con ese más allá espiritual, no se separa de otras posiciones liminales también asociadas a sus intervenciones: la determinación de ese rol viene mediada por un periodo de padecimiento "la enfermedad de la llamada" que se sana con seguir ese destino espiritual en el transcurso del cual tendrá que 
padecer enfermedades para posteriormente entender y curar desde la salud al enfermo. Se mueve entre lo tradicional y la modernidad, siendo referente en un culto flexible al integrar continuamente personas y situaciones vividas (como el hecho de usar objetos y actitudes identificativas o tributar cosas de su agrado a los parientes recién fallecidos, o trasladar lugares de culto a las aldeas tras la reubicación de la población en el periodo socialista). Su hogar y aprendizaje se sitúa en el límite de la aldea o en la cercanía del bosque en el hábitat disperso: entre la naturaleza primigenia que examina y donde desarrolla sus conocimientos botánicos; y las casas y campos domesticados donde realiza las tareas propias de su género y edad como una persona más en la población.

Gran parte de estos roles de curanderos y consejeros son asignados a mujeres, que en una sociedad patrilocal pertenecen a una familia y viven en otra, manteniendo vínculos directos con ambas; pero además la identidad de género que muestran en el día a día, se muestra con ambigüedad en las ceremonias, en las que su propia personalidad puede ser suplantada en la apariencia de cualquier otro antepasado. Esta situación ambigua, en el sentido de apertura a diferentes realidades que otros viven como excluyentes, probablemente se extiende a muchas otras facetas de la vida, o así lo espero seguir comprobando en mis futuras visitas al campo, porque además es parte de la formación inherente a ese conocimiento.

Por tanto, esta capacidad de penetrar y pertenecer sin distorsión a diferentes realidades parece ser lo valorado en los curanderos, obteniendo de esta capacidad de mediación su reconocimiento. En el análisis más detallado sobre el poder por parte de Farré (2008: 404) se describe el poder tradicional materializado en el rito y personificado en las figuras del rey (Hosi) y los curanderos (nyanga y nyamussoro) en una posición que desborda el ámbito social para mediar con las fuerzas de la naturaleza (ntumbuluku). Obviamente, si pensamos en peso absoluto, la dimensión espiritual-religiosa tiene un gran peso por lo inaccesible que resulta una dimensión espiritual paralela reconocida socialmente (así como el exclusivo juego entre la vida y la muerte al que viene asociado). Pero no podemos dejar de lado esas dimensiones sociales que lleva aparejadas, porque de ahí emana el poder de convocatoria, comunicación y retribución entre los que son y los que fueron, que está presente en toda intervención. Esta capacidad de entender integra el conocimiento y la empatía con algo próximo, con la visión más global o desde una cierta distancia, buscando en ello una razón objetiva para las realidades en juego.

Así, se acude al curandero en casos de crisis familiar, cambio (por ejemplo buscar suerte antes de emigrar) tomar decisiones relevantes, buscar justicia o redención, evitar o solucionar problemas, enfermedad... En definitiva para pedir consejo y guía al que se entiende que puede transmitirnos o ayudarnos a alcanzar los medios para llegar a la solución, al que tiene ese poder o ese conocimiento.

Por todo ello, para estudiar esta figura también tenemos que estudiar su rol en las diferentes realidades en que aprende, movernos siguiendo ese dinamismo entre fronteras que dividen mundos y sus parcelaciones, entre las que se encuentra el género, el grupo, el territorio, el tiempo y su parcelación (que establece roles en relación a la edad y el momento). Transitar entre la complejidad de los mundos que conforman las personas como objetos de estudio. Alcanzar el conocimiento desde esa posición híbrida, multisituada en su posición y percepción. Dialogar entre realidades, traducir significados, sentir y razonar para entendernos y cuestionarnos... En definitiva, creo que nuestro papel como antropólogos tiene que ver tanto con ello que no sólo encaja en este rol, sino que parece ser una definición del mismo, incluyendo la consciencia de que su objeto de estudio no es estático y por tanto su observación tampoco ha de serlo.

\section{Conclusiones}

A lo largo del texto se ha intentado incidir en el movimiento, sus hitos y su integración como base de construcción de las sociedades, centrándonos en un caso paradigmático en 
África Austral. La apropiación de la experiencia, del camino, así como la creación, reflexión y comunicación desde los nodos nos introduce en un campo de estudio que transcurre en paralelo en diferentes realidades. Así, de toda esta argumentación, y siendo el mundo virtual un ejemplo de canal pero también de contexto en interacción, se puede concluir que el trabajo de campo debe ser multisituado, al menos por tres razones:

1-Las sociedades se forman en su interacción con/desde diferentes contextos.

2- Los contextos diferentes cambian a los individuos que se mueven en ellos (tanto por sus diferencias como por la experiencia vivencial de transición).

3- Se acotan, transforman e interpretan tanto los lugares como las personas relacionadas con ellos a partir de atribuciones que crean realidades (territorios, paisajes, identidades, identificaciones, conexiones...).

Esa necesidad de analizar el movimiento, las conexiones múltiples y sus reconstrucciones no se detiene únicamente en los lugares físicos sino que, por su transcendencia, debemos prestar atención a las fronteras sociales y simbólicas desde las que se integran perspectivas, roles, funciones, identidades, etc. que componen el dinamismo de la vida social.

Para acabar, me gustaría insistir en ese espacio híbrido muchas veces discriminado pero que nos permite mirar desde la distancia estando dentro, analizar desde la razón empatizando con el sentimiento, ese lugar que no es dicotómico ni radical sino mestizo y que tiene que ver con los prismas múltiples y la visión humilde desde diferentes gafas, en definitiva, con nuestra disciplina y con la forma en que aquí ha quedado definido el conocimiento.

\section{Bibliografía}

Alconada, A. (2013). Ressano García. Villa y frontera. Studia Africana, volumen 24: 107-116.

Bhabha, H.K. (1996). Culture's in between. En: Hall, S., du Gay, P. (Eds.) Questions of cultural identity: 53-60. London, UK: Sage Publications.

Carter, D. (2003) Preface. En: Khalid Koser (Ed.). New African Diaspora: IX-XIX. London and New York: Routledge.

Cátedra, M. (2017). Desde la distancia: aquella joven antropóloga. En: Vicente, T., Albert, M., Espeso, P., Pastor, M.J. (Eds.) Antropologías en transformación: sentidos, compromisos y utopías: 191-209. Valencia, España: Institució Alfons el Magnànim - Centre Valencià d'Estudis i d'Investigació.

Covane, L.A. (2001). O trabalho migratório e a agricultura no Sul de Moçambique (1920-1992). Colecçao Identidades. Maputo, Moçambique: Promédia.

Farré, A. (2008) Vínculos de sangue e estruturas de papel: ritos e territorio na história de Quême (Inhambane). Análise Social, XLIII (2): 393-418.

Felipe, T. (2016). El signo paisaje cultural desde los horizontes de la antropología semiótica. AIBR, vol. 1(1): 105-129.

Goody, J. (1971). The development cycle in domestic groups. UK: Cambridge University Press.

Honwana, A.M. (2003). Espiritos vivos, tradiçoes modernas: posessao de espiritos e reintegraçao social pós-guerra no Sul de Moçambique. Lisboa, Portugal: Ela por Ela.

Kopytoff, I. (1987). The African Frontier. The reproduction of traditional African Societies. Indianapolis, USA: Indiana University Press.

McDonald, D.A. (2000). On borders. Perspectives on international migration in Southern Africa. Kingston, Ontario-Canada: Southern African Migration Project, St. Martin’s Press.

Smith, J.H., Mwadime, N. (2014). Email from Ngeti. Oakland, California-USA: University of California Press.

Sow, Papa (2007). Diásporas africanas y mundialización: de la representación histórica a la toma de conciencia. En: Iniesta, F. (ed.) África en diáspora. Movimientos de población y políticas estatales: 135-150. Barcelona, España: Bellaterra. 